\title{
Radiation Dose from Computed Tomography Scanning in Patients at Songklanagarind Hospital: Diagnostic Reference Levels
}

\author{
Dechen Pema, M.D. ${ }^{1}$, Supika Kritsaneepaiboon, M.D. ${ }^{2}$
}

'Department of Radio-Imaging and Diagnosis, Jigme Dorji Wangchuck National Referral Hospital, Thimphu 11001, Bhutan.

${ }^{2}$ Section of Pediatric Imaging, Department of Radiology, Faculty of Medicine, Prince of Songkla University, Hat Yai,

Songkhla 90110, Thailand.

Received 11 September 2019 • Revised 20 September 2019 • Accepted 25 November 2019 • Published online 27 March 2020

\begin{abstract}
:
Objective: To determine diagnostic reference levels (DRLs) of computed tomography (CT) radiation doses in terms of CT dose index volume $\left(\mathrm{CTDI}_{\mathrm{vol}}\right)$ and dose length product (DLP) of CT scans of the head, chest and abdomen for patients at Songklanagarind Hospital, Thailand.
\end{abstract}

Material and Methods: This was a retrospective analysis of 463 randomly selected head, chest and abdominal CT stuides from 416 patients enrolled from July $1^{\text {st }}$ to $31^{\text {st }}$ 2017. The CTDI $v$, DLP and clinical indication for each CT study were conducted. The median and third quartile values were analysed and compared to the standard international DRLs. The DRL was defined as the third quartile value.

Results: The DRLs for CTDI ${ }_{\text {vol, }}$ and DLP of head, chest and whole abdominal CT were 57.5, 11.6 and 13.1 milliGray (mGy), and 1,102.6, 474.7 and 624.4 milliGray x centimetre (mGy.cm), respectively. The most common clinical indications were stroke (29.1\%) for head CT and malignancy for both chest $(73.6 \%)$ and abdominal CTs $(49.6 \%)$.

Conclusion: The DRLs of each CT region were mostly below standard international DRLs of Australia, Europe, Japan, the United Kingdom and the United States. The clinical indication for malignancy had significant difference in the DLP values than other clinical indications in head and chest CT.

Keywords: computed tomography, CT dose index, dose length product, diagnostic imaging, diagnostic reference level, radiation dose

Contact: Dechen Pema, M.D.

Department of Radio-Imaging and Diagnosis, Jigme Dorji Wangchuck National Referral Hospital, Thimphu 11001, Bhutan.

E-mail: dpema1278@gmail.com

This is an open access article under the CC BY-NC-ND license

(http://www.jhsmr.org/index.php/jhsmr/about/editorialPolicies\#openAccessPolicy).

J Health Sci Med Res 2020;38(2):135-143 doi: $10.31584 /$ jhsmr.2020732 www.jhsmr.org 


\section{Introduction}

Computed tomography (CT) was introduced in 1972 and multi-detector CT (MDCT) came about in 1998. Nowadays, both of these are considered as important diagnostic tools, and there has been an exponential rise in numbers of CT scans performed in the United States (US) of nearly 70 million in $2007 .{ }^{1}$ They are a major source of ionizing radiation, as a 2009 US study found CTs alone (11.0\% X-ray based imaging) being responsible for $75.0 \%$ of effective radiation doses from medical imaging. ${ }^{2}$ Since then medical literature has increasingly focused on the significance of patient radiation doses. ${ }^{3}$ More pediatric patients undergoing CT scans and rapid advancements in CT applications provide the main concern for biological adverse side effects and the risk of future cancers. ${ }^{4-6}$ Although the association of ionizing radiation with subsequent development of cancer was based mainly on data obtained from studies of the Japanese atomic bomb survivors, patients should not be discourage from having appropriate diagnostic imaging with dose optimization. ${ }^{7-11}$

United Nations Scientific Committee on the Effects of Atomic Radiation (UNSCEAR) suggested 10-100 milliGray (mGy) is the absorbed dose for low-LET radiation to an individual from multiple whole body CT scans. ${ }^{12}$ The International Commission on Radiological Protection (ICRP) proposed diagnostic reference levels (DRLS), defined as investigational levels applied to easily measured quantity using standard phantom or representative patient, expressed as volume $\mathrm{CT}$ dose index $\left(\mathrm{CTDI}_{\mathrm{vol}}\right)$, dose length product (DLP) and effective dose $e^{13,14}$ to help optimize radiation doses and identify unjustified doses. ICRP emphasized that DRLs should be derived from national or local data and the "As Low as Reasonably Achievable" or ALARA principle be adhered to so as to minimize potential hazards of ionizing radiation.

There were many countries in Western and Asian regions that have established and reported their national
DRLs for adult CT studies: for example Australia, Northern Greece, Turkey, the United Kingdom (UK), other European countries, US, Iran, Malaysia, Japan, and India. ${ }^{15-25} \mathrm{~A}$ study in Thailand in 2012 found CT radiation dose of head below international DRLs but those of CT chest and abdomen exceeded the international DRLs. ${ }^{26}$ A 2011's study at five Thai university hospitals, including Songklanagarind Hospital, noted DRLs of adult brain, chest and abdominal CT varied widely but were within acceptable levels compared to international DRLs. ${ }^{27}$

The objective of this study was to obtain CT radiation DRLs in terms of CTDI ${ }_{\text {vol }}$ and DLP of the most commonly carried out regions of CT scans for adult patients at Songklanagarind Hospital then to and compare then with both international and prior DRLs.

\section{Material and Methods}

The study was approved by the Human Research and Ethics Committee of the Faculty of Medicine, Prince of Songkla University, REC.: 61-176-7-4.

A retrospective descriptive study with analytic component of 463 CT scans of head, chest and abdomen at Songklanagarind Hospital was conducted. The enrollment period was from July $1^{\text {st }}$ to $31^{\text {st }}, 2017$. Exclusion criteria were an age less than 15 years, contiguous chest- abdomenpelvic CT scans and high-resolution CT (HRCT) of the chest.

A CT scanner of 80 slices manufactured by Toshiba, model Aquilion ${ }^{\mathrm{TM}}$ PRIME with serial number BKA 1522134 installed in 2012 in our unit.

All CT scans of adults, fulfilling inclusion and exclusion criteria during the study period, were assigned serial numbers for a total of 1,136 CT scans. Computer generated random selection of these serial numbers fulfilled the calculated total sample size of 463 as well as the categorized sample size. CT data of only selected serial numbers were manually collected from the Picture Archiving 
and Communications system at the Department of Radiology, Faculty of Medicine, Songklanagarind Hospital, Prince of Songkla University.

Independent variables were demographic data including gender and age of patients and the clinical indications for each CT study. The clinical indications were categorized into trauma, infection/inflammation, malignancy, congenital, stroke and others (miscellaneous). The examples of others category are chronic headaches in head CTs, pleural effusion, sarcoidosis in chest CT, chronic abdominal pain, obstruction, anemia, ureteric stones, aneurysms and myomas in abdominal CTs.

The radiation dose parameters provided from the

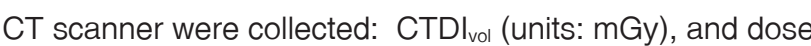
length product (DLP) (units: mGy.cm). The DRL was defined as the $3^{\text {rd }}$ quartile of patient dose distribution (median) for each protocol. A total sample size of 463 was established by equation from available data and further stratified proportionately into the three CT categories. The effective dose (unit milliSievert) which was the biological dose determining the overall long term risk was also calculated by multiplying DLP by conversion co-efficient factor for respective anatomical regions. ${ }^{27}$

The categorical variables such as like demographic data were presented as counts and percentages. Mean \pm standard deviation, median and inter-quartile range (IQR) of the CTDI vol and DLP values were calculated and tabulated. The DRLs obtained by our study were compared to the prior 2011 study at our CT unit and to international DRLs. ${ }^{15,18-21,27}$ Our DRLs and the clinical indications for each CT anatomical region were analyzed using the Kruskal-Wallis rank sum test. A p-value of $<0.05$ was considered to be statistically significant.

\section{Results}

Demographic data and clinical indication distribution according to CT anatomical regions were shown in Table 1 and 2.

The median (interquartile range) DLP in different clinical conditions were shown in Table 3. In head CT, the DLP in patients with malignancy was significantly higher than stroke, trauma, infection/inflammation, congenital anomaly, and other conditions ( $p$-value<0.05). Whereas, the DLP of chest CT or whole abdominal CT in patients with malignancy were not significantly different from another conditions ( $p-$ value>0.05).

The CTDI ${ }_{\text {vol, }}$ DLP and effective dose of head, chest and whole abdominal CT in different phases were shown in Table 4. Numbers of CT studies with CTDI vol and DLP above the third quartile (DRLs) were shown in Table 5.

Table 1 Demographic data of the participants

\begin{tabular}{lllll}
\hline Data & Head CT & Chest CT & Whole abdomen CT \\
\hline No. of patients & 210 & 71 & 135 & 416 \\
No. of CT scans & 254 & 72 & 137 & 463 \\
Male, n (\%) & $98(47.0)$ & $38(54.0)$ & $56(42.0)$ & $192(46.0)$ \\
Age (years), mean \pm S.D. & $57.6 \pm 19.7$ & $59.4 \pm 16.8$ & $54.9 \pm 15.8$ & $57.0 \pm 18.0$ \\
\hline
\end{tabular}

$\mathrm{CT}=$ computed tomography, No.=number, n=number of patients, S.D.=standard deviation 
Table 2 Clinical indication distribution according to computed tomography anatomical regions

\begin{tabular}{llll}
\hline Clinical indications & $\begin{array}{l}\text { Head CT } \\
\text { Number (\%) }\end{array}$ & $\begin{array}{l}\text { Chest CT } \\
\text { Number (\%) }\end{array}$ & $\begin{array}{l}\text { Whole abdomen CT } \\
\text { Number (\%) }\end{array}$ \\
\hline Malignancy & $59(23.0)$ & $12(17.0)^{\star}$ & $68(50.0)$ \\
Stroke & & $41(57.0)^{\star *}$ & N/A \\
Trauma & $74(29.0)$ & - & $12(9.0)$ \\
Infection/Inflammation & $73(29.0)$ & $16(22.0)$ & $42(31.0)$ \\
Congenital anomaly & $15(6.0)$ & $1(1.0)$ & $1(1.0)$ \\
Others & $16(6.0)$ & $2(3.0)$ & $14(10.0)$ \\
\hline
\end{tabular}

*Scan length covering entire chest only

${ }^{* *}$ Scan length covering entire chest+upper abdomen down to inferior pole of right kidney

$\mathrm{CT}=$ computed tomography, N/A=not applicable

Table 3 Comparison of dose length product (mGy.cm) according to clinical indications

\begin{tabular}{llll}
\hline Clinical indications & Head $\mathbf{C T}(\mathbf{m G y . c m})$ & Chest $\mathbf{C T}(\mathbf{m G y . c m})$ & Whole abdomen CT $(\mathbf{m G y . c m})$ \\
\hline Malignancy & $2,067(1,920,2,161)$ & $326(267,474)^{\star}$ & $992(604,1,467)$ \\
& & $357(278,473)^{\star \star}$ & \\
Stroke & $1,048(981,1,103)$ & $\mathrm{N} / \mathrm{A}$ & $\mathrm{N} / \mathrm{A}$ \\
Trauma & $1,069(1,009,1,115)$ & $\mathrm{N} / \mathrm{A}$ & $1,174(914,1,416)$ \\
Infection/inflammation & $1,953(1,033,1,080)$ & $276(258,349)$ & $1,072(649,1,620)$ \\
Congenital anomaly & $1,026(978,1,080)$ & $479($ only 1 case) & 2,247 (only 1 case) \\
Others & $1,322(1,048,2,113)$ & $517(345,679)$ & $1,500(1,221,1,416)$ \\
\hline
\end{tabular}

Data are presented as median (interquartile range)

*Scan length covering entire chest only

**Scan length covering entire chest+upper abdomen down to inferior pole of right kidney

$\mathrm{CT}=$ computed tomography, $\mathrm{N} / \mathrm{A}=$ not applicable

Table 4 Volume computed tomography dose index, dose length product, and effective dose of computed tomography head, chest and whole abdomen

\begin{tabular}{lllll}
\hline Region & Phase $(\mathbf{n})$ & CTDI $_{\text {vol }}(\mathbf{m G y})$ & DLP $(\mathbf{m G y . c m})$ & Effective dose (mSV) \\
\hline Head CT & Non-contrast (186) & $55.8(52.3,57.5)$ & $1,048.7(99.7,1,102.6)$ & $2.5(2.3,5.5)$ \\
& Non-contrast and contrast- & $57.5(52.3,57.5)$ & $2,113.0(1,990.0,2,188.0)$ & $4.9(4.6,5.1)$ \\
& enhanced (68) & & & \\
Total (254) & $55.8(52.3,57.5)$ & $1,089.9(1,009.2,1,924.6)$ & $2.5(2.3,4.4)$ \\
Chest CT & Contrast (72) & $7.9(6.2,11.6)$ & $317.0(260.6,474.7)$ & $5.4(4.4,8.1)$ \\
\hline
\end{tabular}


Table 4 (continued)

\begin{tabular}{|c|c|c|c|c|}
\hline Region & Phase (n) & $\mathrm{CTDI}_{\mathrm{vol}}(\mathrm{mGy})$ & DLP (mGy.cm) & Effective dose (mSV) \\
\hline \multirow[t]{5}{*}{ Whole abdomen CT } & Plain (17) & $9.6(7.8,26.8)$ & $397.8(299.1,488.7)$ & $6.0(4.5,7.3)$ \\
\hline & Arterial (20) & $9.2(7.6,13.0)$ & $324.9(223.9,469.2)$ & $4.8(3.3,7.0)$ \\
\hline & Venous (56) & $9.4(7.7,13.2)$ & $449.2(351.7,624.4)$ & $6.7(5.3,9.4)$ \\
\hline & Delayed (44) & $13.1(9.1,16.5)$ & $340.0(257.2,547.3)$ & $5.1(3.98 .2)$ \\
\hline & Total (137) & $9.4(7.7,13.2)$ & $1,091.7(677.8,1,521.7)$ & $16.4(10.2,22.8)$ \\
\hline
\end{tabular}

Data are presented as median (interquartile range)

$\mathrm{CT}=$ computed tomography, CTDIvol=CT dose index volume, DLP=dose length product, mGy=milliGray (unit for $\mathrm{CTDI}$ vol), mGy.cm=milliGrayx centimetre (unit for DLP), mSV=milliSievert (unit for effective dose), $n=$ number of patients

Table 5 Number of computed tomography studies with computed tomography dose index volume, and dose length product above third quartile (diagnostic reference levels)

\begin{tabular}{llll}
\hline DRLs & Head CT & Chest CT & Whole abdomen CT \\
\hline CTDI $_{\text {vol }}$ (mGy) above third quartile & $>57.5$ & $>11.6$ & $>13.2$ \\
$\mathrm{n}(\%)$ & $63(24.8)$ & $18(24.8)$ & $34(24.8)$ \\
DLP (mGy.cm) above third quartile & $>1,102.6$ & $>474.7$ & $>624.4$ \\
$\mathrm{n}(\%)$ & $54(21.3)$ & $18(25.0)$ & $34(24.8)$ \\
\hline
\end{tabular}

$\mathrm{CT}=$ computed tomography, $\mathrm{CTDlvo}=\mathrm{CT}$ dose index volume, $\mathrm{DRL}=$ diagnostic reference levels, $\mathrm{DLP}=$ dose length product, $\mathrm{mGy}=$ milliGray (unit for CTDIvol), mGy.cm=milliGray $x$ centimetre (unit for DLP), $m S V=$ milliSievert (unit for effective dose), $n=$ number of studies

\section{Discussion}

The rapidly increasing number of CT scans causes more concern for patient radiation doses coupled with their adverse effects. The ICRP proposed DRLs as a way for optimization and justification of CT radiation doses, with emphasis on obtaining DRLs from existing, local data. A study by Najafi et al. $^{22}$ in 2014 of 24 MDCT centers in Iran analyzed 885 reports. In terms of DLP, the DRLs of an adult age group were 700, 290, 330, and 550 mGy. $\mathrm{cm}$ for the head, sinus, chest, abdominal and pelvis CTs respectively. Most cases were less than the international reference values of Australia and European countries. ${ }^{15,19}$ Another study aimed to establish the first DRLs for CT examinations in adult and pediatric patients in Turkey from 167 hospitals found that adult head CT doses and many pediatric CT doses were higher than European Commission recommendation. ${ }^{17} \mathrm{~A}$ few studies suggested to replace alternative imaging examination in clinically non-indicated CT scans or using the tube current modulation to reduce the $\mathrm{CT}$ radiation dose in children or patients undergoing multiple CT scans. ${ }^{28,29}$ A 2011's study in Thailand encouraged CT units including Songklanagarind Hospital to maintain their own DRLs due to the wide variability. ${ }^{27}$ Our study gave the updated $\mathrm{CT}$ radiation data of the CT unit at Songklanagarind Hospital in Table 6 and provided a larger sample population as well as correlation with clinical 
Table 6 Comparison of the diagnostic reference levels in computed tomography imaging of head, chest and whole abdomen

\begin{tabular}{|c|c|c|c|c|c|c|c|}
\hline \multirow{2}{*}{ CT Anatomical region } & \multicolumn{2}{|c|}{$\begin{array}{c}\text { DRLs of } \\
\text { Songklanagarind Hospital }\end{array}$} & \multicolumn{5}{|c|}{ International DRLs } \\
\hline & This study & $\begin{array}{l}\text { Previous } \\
\text { study in } \\
2011^{27}\end{array}$ & $\begin{array}{l}\text { UK } \\
(2011)^{18}\end{array}$ & $\begin{array}{l}\text { EC } \\
(2014)^{19}\end{array}$ & $\begin{array}{l}\text { US } \\
(2017)^{27}\end{array}$ & $\begin{array}{l}\text { Australia } \\
(2013)^{15}\end{array}$ & $\begin{array}{l}\text { Japan } \\
(2015)^{21}\end{array}$ \\
\hline \multicolumn{8}{|l|}{ Head CT } \\
\hline CTDlvol (mGy) & 57.5 & 45.0 & 60.0 & 60.0 & 56.0 & 60.0 & 85.0 \\
\hline DLP (mGy.cm) & $1,102.6$ & $1,089.0$ & 970.0 & 970.0 & 962.0 & $1,000.0$ & $1,350.0$ \\
\hline \multicolumn{8}{|l|}{ Chest CT } \\
\hline CTDIvol (mGy) & 11.6 & 8.6 & 12.0 & 10.0 & 13.0 & 15.0 & 15.0 \\
\hline DLP (mGy.cm) & 474.7 & 355.0 & 610.0 & 400.0 & 469.0 & 450.0 & 550.0 \\
\hline \multicolumn{8}{|l|}{ Whole abdomen CT } \\
\hline CTDIvol (mGy) & 13.2 & 11.3 & 15.0 & 25.0 & 15.0 & 15.0 & 20.0 \\
\hline DLP (mGy.cm) & 624.4 & 552.0 & 745.0 & 800.0 & 755.0 & 700.0 & $1,000.0$ \\
\hline
\end{tabular}

$\mathrm{CT}=$ computed tomography, $\mathrm{CTD} \mathrm{I}_{\mathrm{vol}}=\mathrm{CT}$ dose index volume, $\mathrm{DRL}=$ diagnostic reference levels, $\mathrm{DLP}=\mathrm{dose}$ length product, $\mathrm{EC}=\mathrm{European}$ Commission, $m G y=$ milliGray (unit for CTDlvol), mGy.cm=milliGray $x$ centimetre (unit for DLP), UK=United Kingdom, US=United States

indications. The DRLs from our srtudy were below inter national DRLs, especially Japan. ${ }^{21}$ Head CTs had CTDIvol slightly higher than the $\mathrm{US}^{20}$ and DLP above those of Australia ${ }^{15}, \mathrm{UK}^{18}, \mathrm{EU}^{19}$, and $\mathrm{US}^{20}$. Our DRLs of all three CT regions have increased from the prior study. ${ }^{27}$

Non-contrast head CTs were performed mostly for strokes followed by trauma and malignancy. Most CT scans in other categories had only non-contrast phase. The total effective dose was not high due to a low conversion factor of the non-radiosensitive brain. Additional contrast enhanced venous phase indicated for malignancy and infection/inflammation had twice the dose of non-contrast studies alone as CT parameters and scan lengths were still the same as non-contrast studies.

All chest CT scans had contrast enhanced venous phase with longer scan length of the chest including upper abdomen down to the inferior pole of right kidney. No significant difference among the clinical indications ( $p-$ value $=0.84$ to 0.99 ) in chest CTs may be due to near identical protocols. For example, insignificant difference between the two protocols for malignancy versus others ( $p$-value $=0.84$ and 0.90 , respectively) was due to almost equal scan lengths of both protocols. Whole abdominal CTs had the highest total effective dose due to longer scanning area, high conversion coefficient of many intraabdominal organs with high tissue weighting factors and multiple phases. Both DLP and effective dose of abdominal CTs including all phases was the highest. The venous phase had the highest DLP as it covered whole abdomen whereas other phases did not. No statistically significant differences among clinical indications in abdominal CT were seen.

The CT DRL values in our unit were below DRLs from the $\mathrm{UK}^{18}, \mathrm{EU}^{19}$ and Australia ${ }^{15}$. Head CTs had CTDIvol 
slightly above that of the US ${ }^{20}$ and DLP above the UK ${ }^{18}$, $E U^{19}, U^{20}$ and Australia ${ }^{15}$. The reason for this may be the scan length scanning the patient down until C2 the spine in indications for trauma or others. The DRLs of all three CT regions of our study were lower than those of Japan. ${ }^{21}$ This fact could be significant as international reference levels were derived from Caucasian people. Taking this into consideration more studies in Asian countries needs to be conducted. The DRLs of this study were higher than the prior study of $2011 .{ }^{27}$ Different CT machines, CT parameter settings to obtain diagnostic quality images and rapidly changing protocols were likely causes.

The proportions of CT scans with radiation dose (DLP) above the DRLs were quite high: head CTs (21.3\%), chest CTs (25.0\%) and whole abdominal CTs (24.8\%). A retrospective analysis of CT parameters and clinical indications will help to both identify and address the reasons for preventive high CT radiation doses.

Our study was limited by exclusion of HRCT of the chest and contiguous chest abdominal and pelvic CT scans. Future studies could be inclusive of more anatomical regions, techniques and indications in the rapidly evolving field of medical imaging. A CT dose index registry maintained with regular review of $C T$ studies above DRLs is recommended. Periodic studies to optimize radiation dose as well as a review of CT protocols by radiologists, medical physicists and technicians especially when new hardware or software is installed is required. CT dose on digital display of annual quality control procedures of the CT scanner should be verified by qualified medical physicists.

We also emphasizes clear communication between radiologists and clinicians, in concerns to the patient details and clinical indications, in order to optimize scan parameters, avoid unnecessary scanning phases and recommend alternate options where possible.

\section{Conclusion}

Our study showed the DRLs of CT scans in adults at Songklanagrind Hospital were; CTDI 57.50 mGy, DLP 1,102.60 mGy.cm for head CTs, CTDI 11.63 mGy, DLP 473.10 mGy.cm for chest CTs and CTDI 13.15 mGy, DLP 1,467.00 mGy.cm for abdominal CTs. The most common clinical indications were: stroke (29.1\%) for head CTs and malignancy for both chest $(73.6 \%)$ and whole abdominal (49.6\%) CTs. Our DRLs were mostly below international DRLs of Australia, Europe, Japan, the UK and the US. There were significant differences in the DLP values of indication for malignancy versus other clinical indications in both head CTs and chest CTs.

\section{Acknowledgement}

We would like to thank the staff and mentors from the Departments of Radiology, Faculty of Medicine, Prince of Songkla University for their kind support.

\section{Conflict of interest}

The authors declare that there is no conflict of interest, regarding the publication of this article.

\section{References}

1. Amis ES, Butler PF, Applegate KE, Birnbaum SB, Brateman LF, Hevezi JM, et al. American college of radiology white paper on radiation dose in medicine. J Am Coll Radiol 2007;4: 272-84.

2. Fazel R, Krumholz HM, Wang Y, Ross JS, Chen J, Ting HH, et al. Exposure to low-dose ionizing radiation from medical imaging procedures. N Engl J Med 2009;361:849-57.

3. McLean AR, Adlen EK, Cardis E, Elliott A, Goodhead DT, Harms-Ringdahl $M$, et al. A restatement of the natural science evidence base concerning the health effects of low-level ionizing radiation. Proc Biol Sci 2017;284(1862). doi: 10.1098/ rspb.2017.1070.

4. Berrington de González A, Mahesh M, Kim KP, Bhargavan $M$, Lewis $R$, Mettler $F$, et al. Projected cancer risks from 
computed tomographic scans performed in the United States in 2007. Arch Intern Med 2009;169:2071-7.

5. Royal HD. Effects of low level radiation-what's new? Semin Nucl Med 2008;38:392-402.

6. Power SP, Moloney F, Twomey M, James K, O’Connor OJ, Maher MM. Computed tomography and patient risk: facts, perceptions and uncertainties. World J Radiol 2016;8:902-15.

7. Doss M. Linear no-threshold model may not be appropriate for estimating cancer risk from CT. Radiology 2014;270:307-8.

8. Preston DL, Ron SE, Tokuoka S, Funamoto N, Nishi M, Soda $\mathrm{M}$, et al. Solid cancer incidence in atomic bomb survivors: 1958-1998. Radiat Res 2007;168:1-65.

9. Linet MS, Kim KP, Miller DL, Kleinerman RA, Simon SL, Berrington de Gonzalez A. Historical review of occupational exposures and cancer risks in medical radiation workers. Radiat Res 2010;174:793-808

10. Mettler FA, Bhargavan M, Faulkner K, Gilley DB, Gray JE, Ibbott GS, et al. Radiologic and nuclear medicine studies in the United States and worldwide: frequency, radiation dose, and comparison with other radiation sources 1950-2007. Radiology 2009;253:520-31.

11. Little MP. Cancer and non-cancer effects in Japanese atomic bomb survivors. J Radiol Prot 2009;29:A43-59.

12. Report of the United Nations Scientific Committee on the Effects of Atomic Radiation 2010: Fifty-seventh session, includes scientific report: summary of low-dose radiation effects on health [monograph on the Internet]. New York: United Nations Scientific Committee on the Effects of Atomic Radiation; 2011 [cited 2018 Apr 3]. Available from: http://www. unscear.org/unscear/en/publications/2010/UNSCEAR_ 2010_Report_M.pdf

13. IAEA safety glossary. Terminology used in nuclear safety and radiation protection [monograph on the Internet]. Vienna; International Atomic Energy Agency; 2018 [cited 2019 Feb 15]. Available from: https://www.iaea.org/publications/11098/ iaea-safety-glossary-2018-edition

14. Mettler FA Jr, Huda W, Yoshizumi TT, Mahesh M. Effective doses in radiology and diagnostic nuclear medicine: a catalog. Radiology 2008;248:254-63.

15. Hayton A, Wallace A, Marks P, Edmonds K, Tingey D, Johnston $P$. Australian diagnostic reference levels for multi detector computed tomography. Australas Phys Eng Sci Med 2013;36: 19-26.

16. Hatziioannou K, Papanastassiou E, Delichas M, Bousbouras P. A contribution to the establishment of diagnostic reference levels in CT. Br J Radiol 2003;76:541-5.

17. Ataç GK, Parmaksız A, İnal $T$, Bulur E, Bulgurlu F, Öncü $T$, et al. Patient doses from CT examinations in Turkey. Diagn Interv Radiol Ank Turk 2015;21:428-34.

18. Doses from computed tomography (CT) examinations in the UK - 2011 review [monograph on the Internet]. London: Public Health England; 2011 [cited 2019 Feb 11]. Available from: https://assets.publishing.service.gov.uk/government/ uploads/system/uploads/attachment_data/file/349188/ PHE_CRCE_013.pdf.

19. Radiation Protection No 180. Diagnostic reference levels in thirty-six European countries [monograph on the Internet]. European Commission (EC). Luxembourg: Publication Office of the European Union; 2014 [cited 2019 Feb 11]. Available from: http://ec.europa>files>RP180 part2

20. Kanal KM, Butler PF, Sengupta D, Bhargavan-Chatfield M, Coombs LP, Morin RL. U.S. Diagnostic reference levels and achievable doses for 10 adult CT examinations. Radiology 2017; 284:120-33.

21. Diagnostic Reference Levels Based on latest Surveys in Japan. Japan DRLs 2015 [monograph on the Internet]. Tokyo: The Japan Medical Imaging and Radiological Systems Industries Association and the national Institute of Radiological Sciences; 2015 [cited 2019 Feb 11]. Available from: http:// www.radher.jp/J-RIME/report/DRLhoukokusyoEng.pdf

22. Najafi M, Deevband MR, Ahmadi M, Kardan MR. Establishment of diagnostic reference levels for common multi-detector computed tomography examinations in Iran. Australas Phys Eng Sci Med 2015;38:603-9

23. Livingstone RS, Dinakaran PM. Radiation safety concerns and diagnostic reference levels for computed tomography scanners in Tamil Nadu. J Med Phys 2011;36:40-5.

24. Saravanakumar A, Vaideki K, Govindarajan KN, Jayakumar S. Establishment of diagnostic reference levels in computed tomography for select procedures in Pudhuchery, India. J Med Phys 2014;39:50-5.

25. Karim MKA, Hashim S, Bradley DA, Bakar KA, Haron MR, 
Kayun Z. Radiation doses from computed tomography practice in Johor Bahru, Malaysia. Rad Phys Chem 2016;121:69-74.

26. Ittivisawakul S. An analysis of radiation dose from CT. J Depart Med Serv 2015;40:52-65.

27. Trinavarat $\mathrm{P}$, Kritsaneepaiboon S, Rongviriyapanich $\mathrm{C}$ Visrutaratna P, Srinakarin J. Radiation dose from CT scanning: can it be reduced? Asian Biomedicine 2011;5:13-21.
28. Dougeni E, Faulkner K, Panayiotakis G. A review of patient dose and optimisation methods in adult and paediatric CT scanning. Eur J Radiol 2012;81:665-83

29. Greess $H$, Nömayr A, Wolf $H$, Baum $U$, Lell M, Böwing B, et al. Dose reduction in $\mathrm{CT}$ examination of children by an attenuationbased on-line modulation of tube current (CARE Dose). Eur Radiol 2002;12:1571-6. 